\title{
Network transition security for transmission switching
}

\author{
Shijun TIAN ${ }^{1}$, Xifan WANG ${ }^{1}$, Xiuli WANG ${ }^{1}$, Chengcheng SHAO $^{1} \mathbb{D}$, \\ Rong $\mathbf{Y E}^{2}$
}

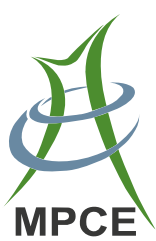

\begin{abstract}
The smart grid with flexible topologies receives intensive attention recently. Transmission switching (TS) alters the power system topology during operation, and has been demonstrated for the advantage of economic and secure operation of power systems. TS includes a chain of sequential switching actions which bring disturbances to the system if the switching actions are not properly designed. Unfortunately, it is not considered or well-studied in existing works. In this paper, a new multi-period TS model that considers the transition security and a two-stage iterative method are proposed. In the TS model, we take into account the fact that only one line is permitted to switch up or down at a time and the security of each switching action is considered. The proposed iterative solution makes the TS model more tractable under AC framework. Case studies on a 6-bus system and the IEEE
\end{abstract}

CrossCheck date: 5 June 2019

Received: 15 October 2018/Accepted: 5 June 2019/Published online: 9 September 2019

(C) The Author(s) 2019

$凶$ Shijun TIAN

tianshijun@stu.xjtu.edu.cn

Xifan WANG

xfwang@mail.xjtu.edu.cn

Xiuli WANG

xiuliw@mail.xjtu.edu.cn

Chengcheng SHAO

ccshao3@xjtu.edu.cn

Rong YE

yerong1985@qq.com

1 State Key Laboratory of Electrical Insulation and Power Equipment, Xi' an Jiaotong University, Xi' an 710049, China

2 State Grid Fujian Power Economic Research Institute, Fuzhou 350012, China 57-bus test system have varified the effectiveness of the proposed model. Numerical results show that: (1) the consideration of transition security of TS is essential; (2) the transition path is directly related to secure and fast the transmission switching; (3) the proposed model and solution method give an effective way to determine the switching sequence and switching timing under transition security criteria.

Keywords Optimization, Security analysis, Topology control, Transmission switching

\section{Introduction}

Conventionally, the branches and bus-bars of transmission network are characterized as fixed assets during system operation. However, as described in [1], "the redundancy built into the transmission network in order to handle a multitude of contingencies over a long planning horizon can, in the short run, increase operating costs". In recent years, the proportion of renewable energies including wind and photovoltaic is growing rapidly on the supply side $[2,3]$. On the load side, the dispatchable loads play more and more important roles in power markets [4]. The stochastic and variable nature of the supply side and load side brings about new operational challenges to power systems. In operational perspective, there usually do not exist a single optimal topology for all periods in the time horizon and/or for all possible market realizations [5]. Therefore, transmission switching (TS) as a new control paradigm is utilized to obtain higher economic and secure benefits by altering the topology of the transmission network during operation. TS has been identified as a 
promising mechanism since it uses existing assets to increase grid flexibility and efficiency [6, 7].

Many works have been devoted to exploiting benefits of TS. The economical generation dispatch incorporated with TS was investigated in [8, 9] in DC framework. Further work was extended to incorporate AC constraints [10-13] and $N-1$ reliability requirements [5, 14]. The co-optimization of generation unit commitment with TS was presented in [15-17]. Besides the studies of decreasing dispatch cost by means of transmission topology control, many other benefits have also been explored, such as voltage stability control [18], congestion management [19, 20], loss reduction [21] and load shed recovery [22]. In addition, researchers analyzed the issuse of vulnerability [23] and stability [24] when TS is implemented.

To manage the computational burden caused by discreteness and nonlinearity, the heuristic algorithms have been investigated to reduce the solution time, such as the space prescreening [25, 26], switchable branch ranking [27], Benders decomposition [14], and parallel computing $[22,28]$.

While these existing studies acknowledge certain benefits of TS, researchers pay little attention to the security and optimality of the transition path from the initial network to the target one. Network reconfiguration represents a chain of time sequential switching operations, considering the fact that the system operators have to operate (switch up or switch down) one line at a time, as shown in Fig. 1. These sequential operations during network transition are similar to contingencies which bring disturbances into the system [29]. Nonetheless, different from forced outages of transmission lines, TS can be implemented in a scheduled way, in which the transition path can be elaborately orchestated by system operators to reduce the disturbances and guarantee the economic and secure operation of power system.

The prevailing framework of switching sequence has been roughly determined empirically or simply based on sensitivity of switching actions to objective function by solving a series of single-line TS problems [8, 14, 22]. The priority list is sorted from the most effective line to the least one in terms of objective that the TS problem

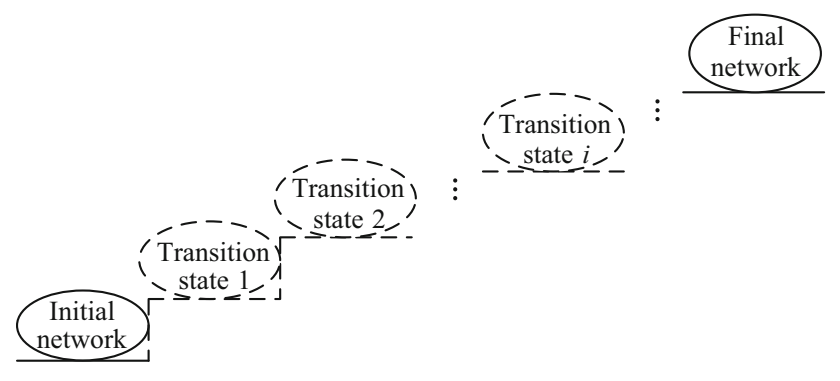

Fig. 1 Step changes of network transition considered. These sensitivity-based algorithms usually provide a feasible solution but not the optimal order of sequential switching.

Furthermore, the existing methods assume that the switching actions operate in a "once every regular time" way. However, it ignores the quick response requirement of TS, which is very important when TS is utilized for restorative or corrective purposes. Also the transition time of TS is expected as short as possible for the consideration of system security and economic efficiency.

To sum up, despite the various benefits brought by TS, there still remain 3 important but suspending problems over transition path of TS before fulfilling automatic intelligent TS:

1) Does switching sequence affect switching security and/or transition time? If so, how to determine the switching sequence?

2) How to determine the switching timings, i.e. time intervals between two switching actions, so as to minimize the duration time of network transition?

3) How to accommodate the generation outputs to ensure transition security?

In this paper, we encapsulated these 3 problems into a multi-period optimal TS model. The model addresses TS along with the optimal transition path in an integrated multi-period problem. The switching actions (up/down) and line states (on/off) are modeled with binary variables. The switching validity constraints are proposed to ensure the principle of "one line switching at a time". The irregular switching timings are modeled by treating time intervals between two switching actions as variables. The power flow constraints before and after each switching operation are included to ensure the switching security considering the contradiction of instant switch between topology and instant consistence of generation outputs when switching action is performed. The standing phase angle difference limit is also considered to ensure the security of rotor shaft impact.

The problem is decomposed and solved in two stages. The first stage is a traditional TS problem that determines the new network topology and the corresponding generation outputs based on specified objective regardless of the transition path and switching security. The second stage is an optimal security transition (OST) problem, which especially addresses the transition path and the security of each switching action based on the results of the preceding TS problem. The OST problem is solved by Benders decomposition method.

The remainder of this paper is organized as follows: the proposed model is formed in Section 2; Section 3 illustrates the decomposition strategy; Section 4 emphasizes the solution of the OST problem using Benders 
decomposition method; and Section 5 presents the case studies. Conclusions are drawn in Section 6.

\section{Problem formulation}

TS process, i.e. network transition, includes a series of switching actions as well as generation re-dispatch, which is depicted as in Fig. 2. Each "s" in red represents one switching action. The whole process is divided into $J^{\prime \prime}+1$ time intervals by $J^{\prime \prime}$ switching actions. Apparently, " $t=0$ " and " $t=J^{\prime \prime}+1$ " represent the initial and final states of the system, respectively. There are basically 3 classes of transition security constraints: power flow constraints, loop closure constraints and ramping constraints. Static security of each switching must be ensured. When switching action happens, the outputs of generation units stay consistent while the power flow changes immediately [21]. In addition, rapid changes are required in the generation dispatch if a line closing causes a loop closure, which could otherwise impact the generator shaft. The corresponding torque induced in the rotor could cause generator fatigue and equipment failure [30]. The standing phase angle difference must be within its limit before an attempt is made to close breakers to protect the rotor shaft. Ramping constraints refer to those the ramping rate of generators should be within their ramping ability.

The multi-period TS considering switching security is formulated as follows:

$$
\begin{aligned}
& \min w_{1}=\sum_{i \in G} c_{i} P G_{i}^{J^{\prime \prime}+1} \\
& \min w_{2}=\sum_{0 \leq t \leq J^{\prime \prime}} T^{t}
\end{aligned}
$$

s.t.

$g\left(P G_{i}^{t}, Q G_{i}^{t-}, z_{k}^{t-1}, V_{i}^{t-}, \delta_{i}^{t-}, S_{k}^{t-}\right)=0$

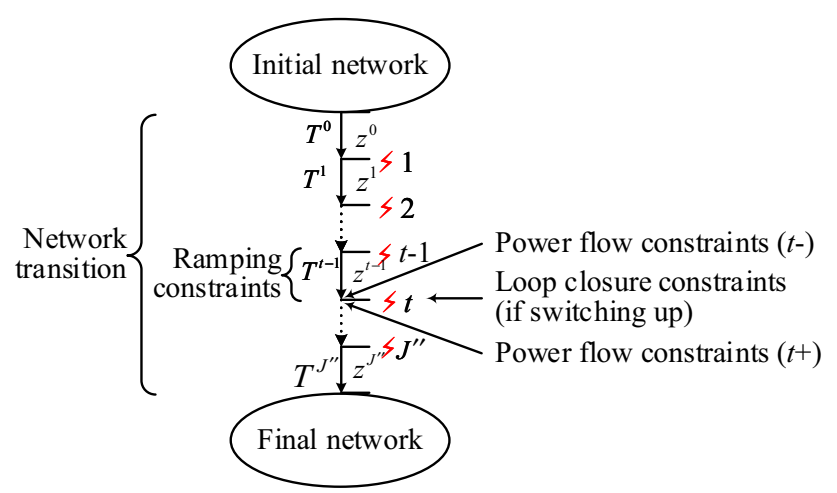

Fig. 2 Network transition and corresponding constraints

$$
\begin{aligned}
& h\left(P G_{i}^{t}, Q G_{i}^{t-}, z_{k}^{t-1}, V_{i}^{t-}, \delta_{i}^{t-}, S_{k}^{t-}\right) \leq h_{\max } \\
& g\left(P G_{i}^{t}, Q G_{i}^{t+}, z_{k}^{t}, V_{i}^{t+}, \delta_{i}^{t+}, S_{k}^{t+}\right)=0 \\
& h\left(P G_{i}^{t}, Q G_{i}^{t+}, z_{k}^{t}, V_{i}^{t+}, \delta_{i}^{t+}, S_{k}^{t+}\right) \leq \eta h_{\max }
\end{aligned}
$$

For (2), (3), (4), (5) $\quad \forall 1 \leq t \leq J^{\prime \prime}+1, k \in K, i \in N$

$z_{k}^{t}-z_{k}^{t-1}=s u_{k}^{t}-s d_{k}^{t} \quad \forall 1 \leq t \leq J^{\prime \prime}, k \in K^{\prime}$

$\sum_{k \in K^{\prime}}\left(s u_{k}^{t}+s d_{k}^{t}\right)=1 \quad \forall 1 \leq t \leq J^{\prime \prime}$

$\delta_{i}^{t-}-\delta_{j}^{t-} \leq \Delta_{k}+M\left(1-s u_{k}^{t}\right) \quad \forall k \in K^{\prime}, 1 \leq t \leq J^{\prime \prime}$

$\delta_{i}^{t-}-\delta_{j}^{t-} \geq-\Delta_{k}-M\left(1-s u_{k}^{t}\right) \quad \forall k \in K^{\prime}, 1 \leq t \leq J^{\prime \prime}$

$T^{t} \geq \mu \quad \forall 1 \leq t \leq J^{\prime \prime}-1$

$\left|P G_{i}^{t}-P G_{i}^{t-1}\right| \leq r_{i} T^{t} \quad \forall 1 \leq t \leq J^{\prime \prime}+1, i \in G$

$\sum_{k \in K^{\prime}}\left|z_{k}^{0}-z_{k}^{J^{\prime \prime}+1}\right|=J^{\prime \prime}$

where $i, j$ are the indexes for buses; $k$ is the index for transmission lines; $t$ is the index for time intervals; $N$ is the set of buses; $G$ is the set of generators; $K, K^{\prime}, K^{\prime \prime}$ represent the set of lines, switchable lines and switching lines (including on and off), respectively; $J^{\prime \prime}$ is the number of switching actions; $P G_{i}, Q G_{i}$ are the active and reactive power generation at bus $i$, respectively; $V_{i}, \delta_{i}$ are the voltage magnitude and angle at bus $i$, respectively; $S_{k}$ is the apparent power flow at line $k ; \eta$ in (6) is the relaxation factor defining how much the short-term security constraints can be temporarily relaxed; Superscript $t$ represents variables corresponding to time interval $t$; " $t-$ " and " $t+$ " mean the variables corresponding to instants before and after each switching action, respectively; $z_{k}^{t}$ is the binary variable which represents the state of line $k$ at time $t(0$ offline, 1-online); $s u_{k}^{t}$ and $s d_{k}^{t}$ are binary variables which represent switch up and down action for line $k$ at time $t$, respectively (0-no switch, 1-switch); $T^{t}$ represents the duration time of time interval $t ; c_{i}$ is the parameter which represents cost of generation located at bus $i ; r_{i}$ is the ramp rate per minute for unit $i ; M$ in (9) and (10) is a big value; $\Delta_{k}$ is the maximum standing phase angle difference of line $k ; \mu$ in (11) is a predefined minimum time.

The first objective (1) indicates that this model is to minimize the overall generation cost of the final network. Objective (2) refers to minimization of the total transition time. This part is especially important when TS is used to achieve restorative or corrective purposes.

Constraints (3) and (4) identify, for each switching operation, the feasibility of the instantaneous state reached just before switching occurrence. Constraints (5) and (6) are analogous to (3), (4) for the post-switching states of 
each switching action. For ease of presentation, we do not present the full formulation of (3)-(6). Constraint (7) is the logic expressions between switching actions and line states. Constraint (8) imposes restrictions that only one switching action is permitted during one timeslot. Constraints (9) and (10) impose standing phase angle difference limits when a line is switched on. Constraint (11) infers that switching time intervals should be larger than a minimum time $\mu$ for the consideration of practical feasibility. Constraint (12) defines maximum allowed ramp of generation outputs. The number of switching actions is restricted to $J^{\prime \prime}$ by (13). Usually, $J^{\prime \prime}$ is predetermined and restricted to a small number due to the reliability of power system.

\section{Decomposition strategy}

If constraints (3)-(6) are formulated in AC framework, the proposed model faces computational challenges. To tackle the intractability of the problem, the proposed model is decomposed into two problems as depicted in Fig. 3. TS problem optimizes the network topology and corresponding generation outputs based on the specified objective and constraints regardless of the transition security. After solving the TS problem, we can obtain the final network topology and corresponding generation outputs. OST problem then inspects to find a secure transition path in the optimal solution provided by the TS module that can satisfy all constraints with the least transition time.

TS problem based on AC formulation has been discussed by some researchers. The objective of TS is usually designed as minimization of overall generation cost, i.e. (1). Such an objective can be easily changed to others as needed. For simplicity, we do not present the formulations of TS in this paper. For more details on TS with AC

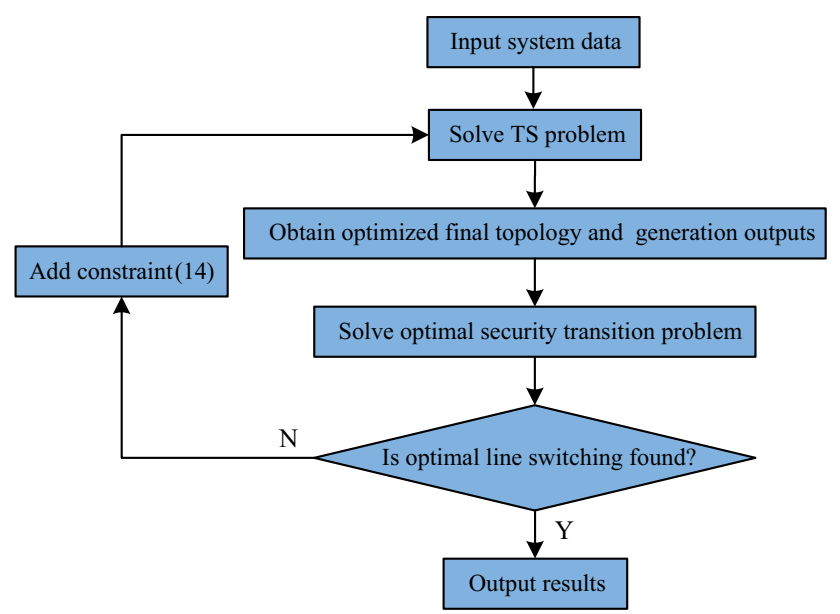

Fig. 3 Flow chart of TS and OST problems security constraints, the interested reader may refer to [13, 14, 31].

The OST problem specially addresses how to transform the system from the original state to the final state securely. The OST model minimizes the total transition time of TS under switching security criteria, i.e. (2). Obviously, the lower bound of the objective is the maximum value of each generation change between initial and final states divided by its ramp rate, i.e. $\max \left(\left|P G_{i}^{J^{\prime \prime}+1}-P G_{i}^{0}\right| / r_{i}\right)$. It happens when all switching actions do not bind any security constraints.

The constraints include (3)-(12), except that all domain expressions $k \in K^{\prime}$ in (7)-(10) are substituted with $k \in K^{\prime \prime}$, since the switching line set $K^{\prime \prime}$ is calculated by preceding TS problem.

There also exits the possibility that: even though both the initial and final networks are securely feasible, the system also faces the potential risk of transitional insecurity, which means that the system fails to survive switching impacts before reaching the final viable operating equilibrium. Under this circumstance, the OST problem is infeasible for particular switching set obtained from TS. Therefore, the infeasible switching set must be cut in the next run of TS by adding constraint (14), as shown in Fig 3.

$\sum_{k \in K^{\prime \prime}}\left|z_{k}^{0}-z_{k}^{J^{\prime \prime}+1}\right| \neq J^{\prime \prime}$

The problem scale especially the number of binary variables in each solving stage decreases drastically by using this decomposition. For a network with $J^{\prime}$ switchable transmission lines and $J^{\prime \prime}$ lines to be switched, the number of binary variables needed in the integrated multi-period TS is $3 J^{\prime} J^{\prime \prime}$, while in decomposed TS and OST problem, the number is $J^{\prime}$ and $3 J^{\prime \prime 2}$ respectively. Usually, $J^{\prime}$ is a relatively large number while $J^{\prime \prime}$ is restricted to a small number in a real power system. Thus, the number of binary variables in each decomposed problem is relatively small, which makes it possible to consider more accurate AC security constraints.

\section{Solution methodology}

The different solution methodologies for TS with $\mathrm{AC}$ constraints have been studied in $[13,14,31]$. In this section, the solution methodology of the OST problem is elaborated. The OST problem is a mixed integer non-linear programming (MINLP) problem. There are mainly two methods to solve MINLP problem. One is based on relaxation [32], such as second-order cone programming (SOCP) relaxation [33]. The other is the widely used 
Benders decomposition. In this paper we choose Benders decomposition to solve the OST problem since it can easily be applied to large-scale systems. It should be noted that the application of both two methods to non-convex MINLP are just heuristic since generalized Benders decomposition assumes convex nonlinear functions when fixing the binary variables, while the power flow equations are nonconvex.

Benders decomposition is designed to separate DC with integer variables as the master problem from AC feasibility check the sub-problem. The master problem determines a rough transition path based on DC constraints. The subproblem checks the AC security of the transition path with the purpose of achieving an AC compatible transition path. Violations are relieved iteratively by adjusting power generation outputs, switching timings and switching sequences.

\subsection{Master problem formulation}

The master problem contains only DC relevant variables, and is formulated as follows:

$$
\begin{aligned}
& \min \sum_{0 \leq t \leq J^{\prime \prime}} T^{t}+\alpha^{*} \\
& \text { s.t. } \\
& g^{\prime}\left(P G_{i}^{t}, z_{k}^{t-1}, \delta_{i}^{t-}\right)=0 \\
& h^{\prime}\left(P G_{i}^{t}, z_{k}^{t-1}, \delta_{i}^{t-}\right) \leq h_{\max }^{\prime} \\
& g^{\prime}\left(P G_{i}^{t}, z_{k}^{t}, \delta_{i}^{t+}\right)=0 \\
& h^{\prime}\left(P G_{i}^{t}, z_{k}^{t}, \delta_{i}^{t+}\right) \leq \eta h_{\max }^{\prime}
\end{aligned}
$$

For (16)-(19) $\forall 1 \leq t \leq J^{\prime \prime}, k \in K, i \in N$

$$
\begin{aligned}
\alpha^{*} & \geq \alpha\left(P G_{i}^{t}, z_{k}^{t}, T^{t}\right) \\
& +\sum_{i \in G} \sum_{1 \leq t \leq J^{\prime \prime}} u_{i}^{t, m-1}\left(P G_{i}^{t}-\widehat{P G}_{i}^{t, m-1}\right) \\
& +\sum_{k \in K^{\prime \prime}} \sum_{1 \leq t \leq J^{\prime \prime}} \pi_{k}^{t, m-1}\left(z_{k}^{t}-\hat{z}_{k}^{t, m-1}\right) \\
& +\sum_{0 \leq t \leq J^{\prime \prime}} \omega^{t, m-1}\left(T^{t}-\hat{T}^{t, m-1}\right)
\end{aligned}
$$

Together with (7), (8), (9), (10), (11), (12), $k \in K^{\prime \prime}$, where $\mu_{i}^{t}, \pi_{k}^{t}$ and $\omega^{t}$ are the marginal values in violation with increase generation for unit $i$ at time $t$, changing in line $k$ at time $t$ and increasing duration time for time interval $t$, respectively; Equations (16)-(19) are the linearized DC constraints of (3)-(6) respectively; $\widehat{P G}_{i}^{t, m-1} \widehat{z}_{k}^{t, m-1}, \hat{T}^{t, m-1}$ are fixed values calculated by master problem at iteration $m-1 ; \alpha$ denotes Benders cuts and corresponds to mismatch at each iteration of sub-problem. In this study, Benders cuts are functions of scheduling variables such as generation outputs $\left(P G_{i}^{t}\right)$, line statuses $\left(z_{k}^{t}\right)$ and time intervals $\left(T^{t}\right)$.

\subsection{Sub-problem formulation}

The sub-problem checks whether the solution obtained from the master problem would lead to violation(s) in AC constraints. If any violation is detected, the related Benders cut will be generated and added to the master problem to recalculate scheduling variables in the next iteration of the master problem. This sub-problem minimizes the total bus power mismatches for all buses and all points of time before and after each switching action, as shown in (21).

$\min \sum_{1 \leq t \leq J^{\prime \prime}} \sum_{i \in N}\left(M P_{i, 1}^{t-}+M P_{i, 2}^{t-}+M P_{i, 1}^{t+}+M P_{i, 2}^{t+}\right)$

where $M P_{i, 1}, M P_{i, 2}$ are surplus and deficit variables. This objective is subject to (7)-(12) with $k \in K^{\prime \prime}$ and AC security constraints (3)-(6) except the modified bus power mismatch equations and generation limits, as shown in (22), (23) and (24), (25), respectively.

$$
\begin{aligned}
& \widehat{P G}_{i}^{t}+M P_{i, 1}^{t-}-M P_{i, 2}^{t-}-P D_{i} \\
& \quad=\sum_{j \in N}\left|V_{i}^{t-}\right|\left|V_{j}^{t-}\right|\left|Y_{i j}^{t-}\right| \cos \left(\delta_{i}^{t-}-\delta_{j}^{t-}-\theta_{i j}^{t-}\right) \\
& \widehat{P G}_{i}^{t}+M P_{i, 1}^{t+}-M P_{i, 2}^{t+}-P D_{i} \\
& \quad=\sum_{j \in N}\left|V_{i}^{t+}\right|\left|V_{j}^{t+}\right|\left|Y_{i j}^{t+}\right| \cos \left(\delta_{i}^{t+}-\delta_{j}^{t+}-\theta_{i j}^{t+}\right) \\
& P G_{i}^{\min } \leq P G_{i}^{t}+M P_{i, 1}^{t-}-M P_{i, 2}^{t-} \leq P G_{i}^{\max } \\
& P G_{i}^{\min } \leq P G_{i}^{t}+M P_{i, 1}^{t+}-M P_{i, 2}^{t+} \leq P G_{i}^{\max } \\
& P G_{i}^{t}=\widehat{P G}{ }_{i}^{t} \leftrightarrow \mu_{i}^{t} \\
& z_{k}^{t}=\hat{z}_{k}^{t} \leftrightarrow \pi_{k}^{t} \\
& T^{t}=\hat{T}^{t} \leftrightarrow \omega^{t}
\end{aligned}
$$

For (22)-(28), $\forall 1 \leq t \leq J^{\prime \prime}, P G_{i}^{\min }, P G_{i}^{\max }$ are the lower and upper limits for active power generation at bus $i$. The arrow in (26)-(28) refers to the duality of the equality constraints. Mathematically, these multipliers represent the marginal increment/decrement of the objective value when corresponding variables change. The iterative Benders procedure stops when the results obtained from the master problem and sub-problem are close enough which can be defined as follows:

$\frac{\left|\alpha^{*, m}-\alpha^{*, m-1}\right|}{\alpha^{*, m-1}} \leq \varepsilon$

It should be noted that, since the master problem is based on lossless DC formation while the sub-problem is based on accurate $\mathrm{AC}$ formation, $P G_{i}^{t}$ in the master 
problem cannot precisely match its value in the AC subproblem. The mismatched gap between DC and AC formation, i.e. the objective (21), are always greater than 0 . Actually, the value of (21) is produced mainly by AC violations at the first few iterations and then converges to system losses as the AC violations are removed iteratively. The algorithm stops when the gap cannot be tightened as iteration number increases. This method may lead to suboptimal solution, but is efficient in finding feasible reliable solutions, as shown in [14], which readers may refer to more information.

\section{Case studies}

We use a six-bus power system and the IEEE 57-bus test system to illustrate the performance of the proposed model and the significance of the transition path. All case studies are solved on a computer with an Intel i5 dual-core processor and 4 GB RAM.

\subsection{Six-bus system}

The six-bus system consists of 2 generation units, 9 transmission lines and 3 loads as shown in Fig. 4. The characteristics of units and lines are elaborated in Tables 1 and 2, respectively. The voltage limit of all buses is restricted to $[0.95,1.05]$ p.u. All transmission lines are closed except Line 3 in the initial network. The result of alternative current optimal power flow (ACOPF) based on the initial network shows that Line 5 is congested. Opening Lines 1, 7 and closing Line 3 are suggested in order to remove congestion by solving TS with AC constraints. Table 3 shows the results of the optimal generation dispatch and overall generation costs (Gen cost) before and after TS.

Then, the transition path is determined by solving the OST problem. The minimum value of the time intervals $\mu$ in (11) is set to 1 minute and the standing phase angle difference limits $\Delta_{k}$ in (9), (10) are set to 5 degrees [30]. The instant line ratings of post-switching is considered to

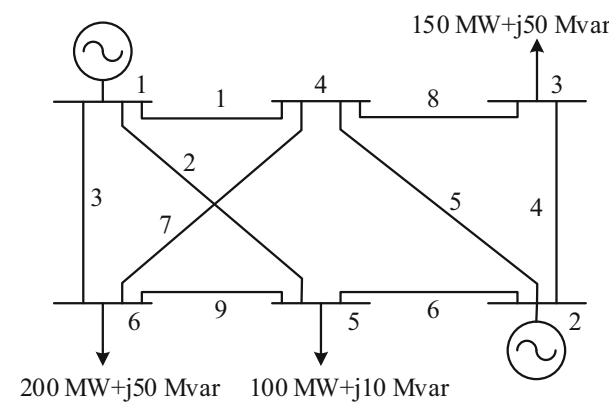

Fig. 4 Six-bus system be $125 \%$ of the normal rating. System loads are treated as constant during transition process. The possible minimum of transition time is 32.96 minutes, which happens when Unit 1 monotonically decreases output at its ramp rate.

By solving the proposed OST problem, we obtain the secure transition path with the switching sequence $7 \rightarrow 1 \rightarrow 3$ and the switching timings at $0,10.31,11.57$, $35.79,53.57 \mathrm{~min}$, respectively. The switching process acts as follows: at $0 \mathrm{~min}$, system operates in initial state, at 10.31, $11.57 \mathrm{~min}$, Line 7 and Line 1 are switched down respectively; at $35.79 \mathrm{~min}$, Line 3 is switched up; the system reaches its new viable steady state at $53.57 \mathrm{~min}$. The corresponding changes of generation over time are depicted as in Fig. 5. Nodal voltage amplitudes during transition process are shown in Fig. 6.

It can be seen that, to meet switching security criteria, generation outputs change non-monotonically: Unit 1 increases before any switching action is taken, then goes down monotonically after Line 7 is switched down; Unit 2 moves oppositely for the reason of power balance. The fluctuating profile of total generation cost is similar to Unit 1. The reason why Unit 1 as well as generation cost increases in the first few minutes is that the system has to transfer to a new temporary status to meet security requirement of the first switching action, i.e. switching down Line 7. The non-monotonic changes of power outputs lead to a transition time of $53.57 \mathrm{~min}, 20 \mathrm{~min}$ longer than the possible minimum transition time.

The nodal voltage amplitudes fluctuate during transition process. From Fig. 6, it can be seen that each switching action changes nodal voltage amplitude instantaneously. Due to the existence of the transition security constraints, all voltage amplitudes are in required security range.

To demonstrate how security constraints affect the transition path of TS, we consider 4 constraint cases for all enumerated permutations of switching sequence. The objective values of each permutation under each case are shown in Table 4, where '-' means infeasible problem. The details of the 4 cases are:

Case 1: Do NOT consider instant post-switching violations and standing phase angle limit constraints;

Case 2: Do NOT consider standing phase angle limit constraints;

Case 3: Consider all of the constraints;

Case 4: Consider all of the constraints, change the flow limit of Line 9 to 200 MVA.

In Case 1, we ignore instant post-switching violation constraints (5), (6) and standing phase angle limit constraints (9), (10). Under this case, 2 out of 6 switching sequences, $1 \rightarrow 3 \rightarrow 7$ and $1 \rightarrow 7 \rightarrow 3$, are infeasible, because opening Line 1 first causes voltage violations on Bus 1 and Bus 3 no matter how generation units 
Table 1 Characteristics of generation units of six-bus system

\begin{tabular}{lllllll}
\hline Unit No. & $\begin{array}{l}P G^{\min } \\
(\mathrm{MW})\end{array}$ & $\begin{array}{l}P G^{\max } \\
(\mathrm{MW})\end{array}$ & $\begin{array}{l}Q G^{\mathrm{min}} \\
(\mathrm{Mvar})\end{array}$ & $\begin{array}{l}Q G^{\max } \\
(\text { Mvar })\end{array}$ & Ramp rate (MW/min) & Cost coefficient (\$/MWh) \\
\hline 1 & 0 & 300 & -150 & 200 & 6 & 40 \\
2 & 0 & 450 & -100 & 250 & 9 & 20 \\
\hline
\end{tabular}

Table 2 Characteristics of transmission lines of six-bus system

\begin{tabular}{llllll}
\hline Line No. & From bus & To bus & $R$ (p.u.) & $X$ (p.u.) & Rate (MVA) \\
\hline 1 & 1 & 4 & 0.02 & 0.1 & 500 \\
2 & 1 & 5 & 0.01 & 0.06 & 500 \\
3 & 1 & 6 & 0.04 & 0.2 & 500 \\
4 & 2 & 3 & 0.02 & 0.1 & 150 \\
5 & 2 & 4 & 0.005 & 0.03 & 100 \\
6 & 2 & 5 & 0.02 & 0.08 & 500 \\
7 & 4 & 6 & 0.02 & 0.08 & 500 \\
8 & 4 & 3 & 0.01 & 0.04 & 500 \\
9 & 6 & 5 & 0.005 & 0.02 & 500 \\
\hline
\end{tabular}

Table 3 Dispatch results for pre and post TS of six-bus system

\begin{tabular}{llll}
\hline State & $P G_{1}(\mathrm{MW})$ & $P G_{2}(\mathrm{MW})$ & Gen cost $(\$ / \mathrm{h})$ \\
\hline Initial & 218.84 & 240.71 & 13567.9 \\
Final & 21.09 & 450 & 9843.5 \\
\hline
\end{tabular}

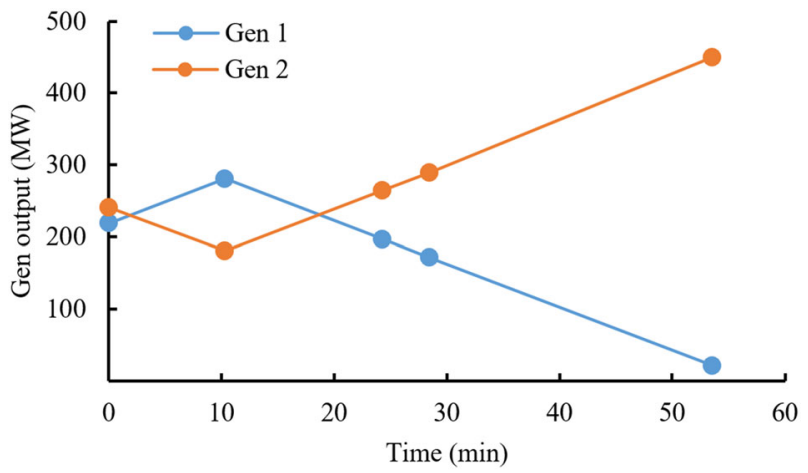

Fig. 5 Changes of generation output over time during system transition of six-bus system

accommodate their outputs. Sequence $3 \rightarrow 1 \rightarrow 7$ is feasible but substantial generating fluctuations are forced to meet security constraints during TS, leading transition time lasts nearly 2 times than other paths.

In Case 2, constraints (5), (6) are added, which means the contradiction of instant change of topology and instant consistence of generation outputs is considered. It can be

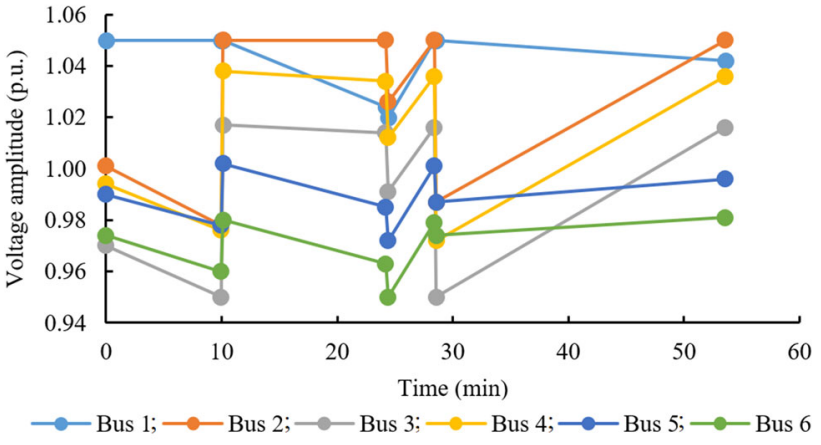

Fig. 6 Changes of nodal voltage amplitude over time during system transition of six-bus system

Table 4 Transition time of different switching sequence under 4 cases

\begin{tabular}{lllll}
\hline Sequence & Case 1 & Case 2 & Case 3 & Case 4 \\
\hline $1 \rightarrow 3 \rightarrow 7$ & - & - & - & - \\
$1 \rightarrow 7 \rightarrow 3$ & - & - & - & - \\
$3 \rightarrow 1 \rightarrow 7$ & 56.96 & - & - & - \\
$3 \rightarrow 7 \rightarrow 1$ & 34.54 & 35.37 & - & - \\
$7 \rightarrow 1 \rightarrow 3$ & 32.96 & 53.57 & 53.57 & - \\
$7 \rightarrow 3 \rightarrow 1$ & 32.96 & 53.57 & - & - \\
\hline
\end{tabular}

seen from Table 4 that instant post-switching constraints significantly affect transition paths. Sequence $3 \rightarrow 1 \rightarrow 7$ fails to meet switching security constraints. For other switching sequences, adding these constraints leads to longer transition time, because generation output strategies have to change to satisfy the new constraints. For example, the post-switching constraints lead to about $20 \mathrm{~min}$ (1.6 times) longer in sequences $7 \rightarrow 1 \rightarrow 3$ and $7 \rightarrow 3 \rightarrow 1$ than that in Case 1. This case indicates that suitable arrangement of transition path can make the whole transition process spend the least time within the system security criteria, while unsuitable arrangement may lengthen the transition time or even jeopardize system security.

The influence of the standing phase angle difference limits on transition path is examined in Case 3. Imposing this limit, when the absolute value of the phase angle difference between two buses of the switched line is larger 
than its standing phase angle difference limit, the line cannot be switched back to the system. The phase angle difference between Bus 1 and Bus 6 is 6.15 degree in the initial state. Standing phase angle difference limit constraint prunes two switching sequences, leaving only one, $7 \rightarrow 1 \rightarrow 3$, that passes all security constraints. It can be found easily that if we relax the standing phase angle difference limit larger than 6.15 degrees, the best switching strategy would be $3 \rightarrow 7 \rightarrow 1$, for it uses only 35.37 minutes to complete system transition.

In Case 4, we change the rating of Line 9 in Table 2 to 200 MVA. In this case, opening Line 7 first leaves only Line 9 to feed the load demand at Bus 6, which would cause load shed because of the transmission flow limit. As shown in Table 4, this case reveals an astonishing result that even the initial and the final networks can sustain feasible operating states, there exist no feasible transition paths that can securely transfer from initial to final network. The feasibility of initial and final operation security does not necessarily mean the feasibility of sequential switching security.

If there exists no feasible transition path like Case 4, constraint (14) is added in the next run of TS problem to remove the infeasible switching set, as shown in Fig. 3. For Case 4, constraint (14) can be specified as follows:

$$
\begin{aligned}
& \left(1-z_{1}\right)+\left(z_{3}-0\right)+\left(1-z_{7}\right) \leq 2 \\
& \left(1-z_{1}\right)+\left(z_{3}-0\right)+\left(1-z_{7}\right) \geq 4
\end{aligned}
$$

Adding (30) and (31), TS problem gives a new network topology with Line 5 and Line 7 opened and Line 3 closed. The generation outputs are $98.9 \mathrm{MW}, 369.3 \mathrm{MW}$, respectively. By solving OST problem, the optimal transition path with the switching sequence is $5 \rightarrow 3 \rightarrow 7$ and the transition time is $31.52 \mathrm{~min}$. Comparison between TS without and with transition security constraints are listed in Table 5. It shows that the the feasibility of final network does not necessarily guarantee the feasibility of transition security. Although the new network is less cost-effective compared to the previous network, the new final network is transitionally feasible.

\subsection{IEEE 57-bus system}

A modified IEEE 57-bus system is also used to study the proposed model. The system has 7 units, 80 branches, and a base load of 1250.8 MW. System data are provided in MATPOWER [34]. Since the data do not specify transmission line flow limits, we assigned the line ratings of Lines 1, 2, 6, 14, 15, 16, 26 to 240 MVA, Lines 3, 7, 18, 22, 25,27 to 100 MVA and others to 60 MVA. The unit ramp rates and bid information are given in Table 6. Furthermore, Line 22 is assumed to be open in the initial network, and the standing phase angle difference limit is set to 5 degrees.

First, the initial network has an optimal overall generation cost of $16040.8 \$ / \mathrm{h}$. By solving TS, we obtain the new network with Lines 27, 28, 6 opened, Line 22 closed, with the optimal overall cost of $15290.7 \$ / \mathrm{h}$. Table 7 shows the results of optimal generation dispatch before and after TS. Then, the OST problem gives the transition path: switching sequence, $27 \rightarrow 28 \rightarrow 22 \rightarrow 6$, switching timings at $0,0.64,1.64,13.66,18.60,21.66 \mathrm{~min}$, respectively. The corresponding generation outputs over time are depicted as in Fig. 7.

It is derived from (12) that monotonic change of unit output contributes to shorter transition time. However, it can be seen from Fig. 7 that most generators change their outputs non-monotonically. The generaton outputs of Units $1,3,6,8$ fluctuate to reach their new states. Unit 9 and Unit 12 change up and down although the final states are the

Table 6 Generation unit information of IEEE 57-bus system

\begin{tabular}{lllll}
\hline Gen No. & $\begin{array}{l}S B P \\
(\$ / \mathrm{MWh})\end{array}$ & $\begin{array}{l}P G^{\mathrm{max}} \\
(\mathrm{MW})\end{array}$ & $\begin{array}{l}P G^{\mathrm{min}} \\
(\mathrm{MW})\end{array}$ & $\begin{array}{l}\text { Ramp rate } \\
(\mathrm{MW} / \mathrm{min})\end{array}$ \\
\hline 1 & 10 & 575.88 & 115.2 & 11.5 \\
2 & 20 & 100 & 20 & 5 \\
3 & 13 & 140 & 28 & 7 \\
6 & 17 & 100 & 20 & 5 \\
8 & 15 & 550 & 110 & 11 \\
9 & 20 & 100 & 20 & 5 \\
12 & 11 & 410 & 82 & 8.2 \\
\hline
\end{tabular}

Table 7 Dispatch results on initial and final networks of IEEE 57-bus system

\begin{tabular}{lllllllll}
\hline State & G1 & G2 & G3 & G6 & G8 & G9 & G12 & Cost $(\$ / \mathrm{h})$ \\
\hline Initial & 280.1 & 20 & 123 & 47.4 & 368.4 & 20 & 410 & 16040.8 \\
Final & 512.5 & 20 & 28 & 38.2 & 256.2 & 20 & 410 & 15290.7 \\
\hline
\end{tabular}

Table 5 Comparison of TS without and with transition security constraints under Case 4

\begin{tabular}{lclll}
\hline Condition & Gen cost $(\$ / \mathrm{h})$ & Switching strategy & Best switching sequence & Transition time $(\mathrm{min})$ \\
\hline Original system & 13567.9 & - & - & - \\
TS without transition security constraints & 9843.5 & Switch down 1,7; Switch up 3 & Non-existent & Non-existent \\
TS with transition security constraints & 11340.5 & Switch down 5,7; Switch up 3 $5 \rightarrow 3 \rightarrow 7$ & 31.52 \\
\hline
\end{tabular}




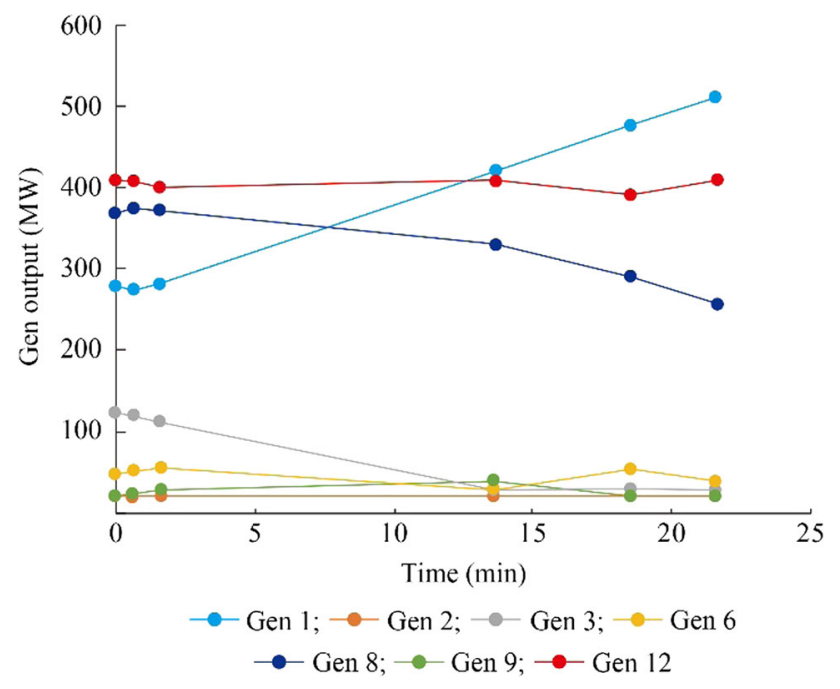

Fig. 7 Changes of generation outputs over time during system transition of the IEEE 57 bus system

same as their initial states. Only one generator, Unit 2, keeps unchanged during transition process. Similarly, generation cost decreases non-monotonically during switching steps. The reason for the non-monotonicity is that generation outputs have to adjust in each switching action to meet the switching security constraints.

The heuristic sensitivity-based algorithm is also tested here. This method determines the switching sequence by solving a series of TS problems. In each TS problem, only one switching action is permitted. The switching sequence obtained by this method is $27 \rightarrow 28 \rightarrow 21 \rightarrow 22$ (the maximum number of actions is limited to 4 ), with the optimal generation cost of $15301.7 \$ / \mathrm{h}$. Although the total cost is only $10 \$ / \mathrm{h}$ higher than the proposed method, these two methods result in two different final networks. In some particular systems these two methods may lead to quite different results. Because the heuristic method chooses each subsequent switching line based on the preceding network rather than in a global optimization way, which gives a possibly suboptimal solution. Also, this method does not take instant post-switching security into consideration. Furthermore, the heuristic method determines only the switching sequence, but does not give the switching timing.

\section{Conclusion}

Nowadays the power system is facing more and more challenges due to the uncertainty and randomness brought by renewable energy resources. TS as a new control paradigm has been demonstrated to solve these problems. This paper copes with network transition security of the TS problem, which has seldom been paid attention to before. The main work and findings are as follows:

1) This paper develops a multi-period transmission switching model considering the network transition security.

2) The problem is solved under a two-stage iterative method under AC framework in which a traditional TS problem provides final network on the first stage and a new OST problem optimizes transition path on the second stage.

3) Numerical results show that: (1) the security of final network cannot guarantee the security of transitional network; (2) switching sequence is highly relevant to transition security and transition time; (3) the consideration of network transition security in TS may lead to a totally different final network compared to TS without network transition security constraints.

Future research will examine the inevitable transient stability of TS.

Acknowledgements This work was supported by National Natural Science Foundation of China (No. U1766205), and Science and Technology Foundation of SGCC "Research on Efficient Integration of Large Scale Long Distance Offshore Wind Farm and Its Key Technologies in Operation and Control".

Open Access This article is distributed under the terms of the Creative Commons Attribution 4.0 International License (http:// creativecommons.org/licenses/by/4.0/), which permits unrestricted use, distribution, and reproduction in any medium, provided you give appropriate credit to the original author(s) and the source, provide a link to the Creative Commons license, and indicate if changes were made.

\section{References}

[1] Hedman KW, Oren SS, O'neill RP (2012) Flexible transmission in the smart grid: optimal transmission switching. In: Sorokin A, Rebennack S, Pardalos PM, Iliadis NA, Pereira MVF (eds) Handbook of networks in power systems I. Springer, Berlin, pp 523-553

[2] Abedinia O, Amjady N (2016) Net demand prediction for power systems by a new neural network-based forecasting engine. Complexity 21(S2):296-308

[3] Abedinia O, Raisz D, Amjady N (2017) Effective prediction model for Hungarian small-scale solar power output. IET Renew Power Gener 11(13):1648-1658

[4] Chen S , Liu CC (2017) From demand response to transactive energy: state of the art. J Mod Power Sys Clean Energy 5(1):10-19

[5] Hedman KW, O'neill RP, Fisher EB et al (2009) Optimal transmission switching with contingency analysis. IEEE Trans Power Syst 24(3): 1577-1586

[6] Hedman KW, Oren SS, O'neill RP (2011) A review of transmission switching and network topology optimization. In: Proceedings of the 2011 IEEE PES general meeting, Detroit, USA, 24-28 July 2011, 7 pp 
[7] Hong H, Hu Z, Guo R, et al (2017) Directed graph-based distribution network reconfiguration for operation mode adjustment and service restoration considering distributed generation. J Mod Power Sys Clean Energy 5(1):142-149

[8] Fisher EB, O'neill RP, Ferris MC (2008) Optimal transmission switching. IEEE Trans Power Syst 23(3):1346-1355

[9] Barrows C, Blumsack S (2012) Transmission switching in the RTS-96 test system. IEEE Trans Power Syst 27(2):1134-1135

[10] Potluri T, Hedman KW (2012) Impacts of topology control on the ACOPF. In: Proceedings of the 2012 IEEE PES general meeting, San Diego, USA, 22-26, July 2010, 7 pp

[11] Soroush M, Fulle JD (2014) Accuracies of optimal transmission switching heuristics based on DCOPF and ACOPF. IEEE Trans Power Syst 29(2):924-932

[12] Khodaei A, Shahidehpour M (2012) Security-constrained transmission switching with voltage constraints. Int J Electr Power Energy Syst 35(1):74-82

[13] Barrows C, Blumsack S, Hines P (2014) Correcting optimal transmission switching for AC power flows. In: Proceedings of 2014 47th Hawaii international conference on system sciences (HICSS), Hawaii, USA, 6-9 January 2014, 6 pp

[14] Khanabadi M, Ghasemi H, Doostizadeh M (2013) Optimal transmission switching considering voltage security and $n-1$ contingency analysis. IEEE Trans Power Syst 28(1):542-550

[15] Hedman KW, Ferris MC, O'neill RP et al (2010) Co-optimization of generation unit commitment and transmission switching with $n-1$ reliability. IEEE Trans Power Syst 25(2):1052-1063

[16] Khodaei A, Shahidehpour M (2010) Transmission switching in security-constrained unit commitment. IEEE Trans Power Syst 25(4):1937-1945

[17] Han J, Papavasiliou A (2016) The impacts of transmission topology control on the European electricity network. IEEE Trans Power Syst 31(1):496-507

[18] Guo WM, Wei Q, Liu GJ et al (2013) Transmission switching to relieve voltage violations in low load period. In: Proceedings of the 2013 4th innovative smart grid technologies Europe (ISGT EUROPE), Lyngby, Denmark, 6-9 October 2013, 5 pp

[19] Nasrolahpour E, Ghasemi H, Khanabadi M (2012) Optimal transmission congestion management by means of substation reconfiguration. In: Proceedings of the 2012 20th Iranian conference on electrical engineering (ICEE), Tehran, Iran, 10-14 May 2012, 6 pp

[20] Khanabadi M, Ghasemi H (2011) Transmission congestion management through optimal transmission switching. In: Proceedings of the 2011 PES general meeting, Detroit, USA, 24-28 July 2011,5 pp

[21] Fliscounakis S, Zaoui F, Simeant G et al (2007) Topology influence on loss reduction as a mixed integer linear programming problem. In: Proceedings of 2007 power tech conference, Lausanne, Switzerland, 1-5 July 2007, 14 pp

[22] Escobedo AR, Moreno-Centeno E, Hedman KW (2014) Topology control for load shed recovery. IEEE Trans Power Syst 29(2):908-916

[23] Zhao L, Zeng B (2013) Vulnerability analysis of power grids with line switching. IEEE Trans Power Syst 28(3):2727-2736

[24] Huang GM, Wang W, An J (2014) Stability issues of smart grid transmission line switching. IFAC Proc Vol 47(3):7305-7310

[25] Liu C, Wang J, Ostrowski J (2012) Heuristic prescreening switchable branches in optimal transmission switching. IEEE Trans Power Syst 27(4):2289-2290

[26] Barrows C, Blumsack S, Bent R (2012) Computationally efficient optimal transmission switching: solution space reduction. In: Proceedings of the 2012 IEEE PES general meeting, San Diego, California, USA, 22-26, July 2010, 8 pp
[27] Fuller JD, Ramasra R, Cha A (2012) Fast heuristics for transmission-line switching. IEEE Trans Power Syst 27(3): 1377-1386

[28] Papavasiliou A, Oren SS, Yang Z et al (2013) An application of high performance computing to transmission switching. In: Proceedings of the 2013 bulk power system dynamics and control: IX optimization, security and control of the emerging power grid (IREP), Rethymnon, Greece, 25-27 August 2013, 6 $\mathrm{pp}$

[29] Liu C, Wang J, Ostrowski J (2012) Static switching security in multi-period transmission switching. IEEE Trans Power Syst 27(4):1850-1858

[30] Nasrolahpour E, Ghasemi H (2015) Congestion management through rotor stress controlled optimal transmission switching. IET Gener Transm Distrib 9(12):1369-1376

[31] Poyrazoglu G, Oh H (2015) Optimal topology control with physical power flow constraints and $n-1$ contingency criterion. IEEE Trans Power Syst 30(6):3063-3071

[32] Hijazi H, Coffrin C, Hentenryck PV (2016) Convex quadratic relaxations for mixed-integer nonlinear programs in power systems. Math Program Comput 9(3):321-367

[33] Kocuk B, Dey SS, Sun X (2017) New formulation and strong MISOCP relaxations for AC optimal transmission switching problem. IEEE Trans Power Syst 32(6):4161-4170

[34] Zimmerman RD, Murillo-Sanchez CE, Thomas RJ (2009) MATPOWER's extensible optimal power flow architecture. In: Proceedings of the 2009 IEEE PES general meeting, Calgary, Canada, 26-30 July 2009, 7 pp

Shijun TIAN received the B.S. degree in electrical engineering from China University of Petroleum, Qingdao, China, in 2011. He is currently pursuing the $\mathrm{Ph}$.D. degree in Xi' an Jiaotong University. His research interests include optimization and control of power system.

Xifan WANG received the B.S. degree from Xi'an Jiaotong University, Xi'an, China, in 1957. From 1983 to 1986, he worked as a visiting scientist at Cornell University, Ithaca, NY, USA. From 1991 to 1994 , he worked as a visiting professor at Kyushu Institute of Technology, Japan. He is currently a member of the Chinese Academy of Science and working as the director of Power System Research Institute in Xi' an Jiaotong University. His research interests include the market, analysis, operation and planning of power systems and novel transmission schemes.

Xiuli WANG received the B.S., M.S., and Ph.D. degrees in electrical engineering from Xi' an Jiaotong University, Xi'an, China, in 1982, 1985, and 1997, respectively. She is currently a Professor at the School of Electrical Engineering, Xi'an Jiaotong University. Her research interests include power market, reliability assessment of power system, and integration of renewable power.

Chengcheng SHAO received the B.S. and Ph.D. degree in electrical engineering from Xi'an Jiaotong University, Xi'an, China, in 2011 and 2017. He is currently an Assistant Professor at Xi' an Jiaotong University. His research interests include the integration of wind power and load dispatch.

Rong YE received the Ph.D. degree in South China University of Technology, Guangzhou, China. His research interests include optimization and control of power system. 